\title{
Interaction of non-parenchymal hepatocytes in the process of hepatic fibrosis (Review)
}

\author{
QI-NI CHENG ${ }^{1-3}$, XUE YANG $^{1-3}$, JIANG-FENG WU $^{1-4}$, WEN-BING AI $^{5}$ and YI-RAN NI ${ }^{1,2}$ \\ ${ }^{1}$ Medical College, China Three Gorges University; ${ }^{2}$ Institute of Organ Fibrosis and Targeted Drug Delivery, \\ China Three Gorges University; ${ }^{3}$ Hubei Key Laboratory of Tumor Microenvironment and Immunotherapy, \\ China Three Gorges University, Yichang, Hubei 443002; ${ }^{4}$ The People's Hospital of China Three Gorges University, \\ Yichang, Hubei 443000; ${ }^{5}$ The Yiling Hospital of Yichang, Yichang, Hubei 443100, P.R. China
}

Received October 3, 2020; Accepted March 2, 2021

DOI: $10.3892 / \mathrm{mmr} .2021 .12003$

\begin{abstract}
Hepatic fibrosis (HF) is the process of fibrous scar formation caused by chronic liver injury of different etiologies. Previous studies have hypothesized that the activation of hepatic stellate cells (HSCs) is the central process in HF. The interaction between HSCs and surrounding cells is also crucial. Additionally, hepatic sinusoids capillarization, inflammation, angiogenesis and fibrosis develop during HF. The process involves multiple cell types that are highly connected and work in unison to maintain the homeostasis of the hepatic microenvironment, which serves a key role in the initiation and progression of HF. The current review provides novel insight into the intercellular interaction among liver sinusoidal endothelial cells, HSCs and Kupffer cells, as well as the hepatic microenvironment in the development of HF.
\end{abstract}

\section{Contents}

1. Introduction

2. LSECs regulate the hepatic microenvironment, activate HSCs and promote HF

3. Activated HSCs interact with LSECs to regulate the hepatic microenvironment

4. Macrophages serve a dual role in HF and participate in pathological angiogenesis

5. Other non-parenchymal hepatocytes also contribute to HF

6. Conclusions

Correspondence to: Dr Yi-Ran Ni, Medical College, China Three Gorges University, 8 Daxue Road, Xiling, Yichang, Hubei 443002, P.R. China

E-mail: nyr1986@163.com

Dr Wen-Bing Ai, The Yiling Hospital of Yichang, 31 Donghu Road, Yiling, Yichang, Hubei 443100, P.R. China

E-mail: 1043642574@qq.com

Key words: hepatic stellate cell, liver sinusoidal endothelial cell, Kupffer cell, hepatic microenvironment, intercellular interaction

\section{Introduction}

Hepatic fibrosis (HF) is a common pathological process that occurs during the development of various chronic liver diseases, such as liver cirrhosis, which initiates a series of biochemical and biophysical changes in the hepatic microenvironment $(1,2)$. It is characterized by a radial linear deposition of collagen around a single hepatocyte and in the space of Disse (3). The progression and regression of HF also relies on a complex interplay of the hepatic microenvironment (4). Hepatic sinusoids provide a biochemical environment for the survival and communication of non-parenchymal hepatocytes, including liver sinusoidal endothelial cells (LSECs), hepatic stellate cells (HSCs) and Kupffer cells (KCs) (5). Intercellular interactions among non-parenchymal hepatocytes are considered to serve a pivotal role in maintaining normal liver function and structure $(6,7)$.

Hepatic sinusoids are specialized capillaries located between liver plates. The wall of the hepatic sinusoid is composed of LSECs, which are distinctive micro-vascular cells that are key to the regulation of the liver microenvironment and represent a permeable barrier to maintain HSC quiescence (8). Under normal circumstances, differentiated LSECs are characterized by the presence of fenestrations and the absence of basement membrane (BM). The fenestration is the most characteristic structure of LSECs and renders the cells highly permeable (9). It also enables the bidirectional transport of metabolites between the circulation and liver parenchyma (10). Actin is the cytoskeletal component of LSECs, which participates in the contraction and expansion of the fenestrae (11). The narrow space between LSECs and liver plate is known as the space of Disse, which contains the extracellular matrix (ECM) and separates sinusoidal cells from parenchymal cells (5). HSCs are specialized pericytes with lipid and retinoid droplets in the cytoplasm that are located in the subendothelial area of hepatic sinusoids. They directly communicate with hepatocytes and LSECs (12). KCs are broadly located in hepatic sinusoids and have an irregular morphology, protruding into hepatic sinusoids or remaining unattached $(13,14)$. There are a number of pseudopods on the surface of KCs, which attach to LSECs or extend into the space of Disse through 
the fenestrations, and directly interact with hepatocytes and HSCs (15).

When the normal hepatic microenvironment is destroyed, the balance between parenchymal hepatocytes and stromal cells is disturbed, leading to the capillarization of LSECs, which is also known as de-differentiation (8). This process increases intrahepatic blood flow resistance and provides a pathological basis for portal hypertension (16). Hepatic sinusoidal capillarization is characterized by the lack of fenestration and formation of organized BM. It is not only a precursor of $\mathrm{HF}$, but also promotes HSC activation. Therefore, the disordered LSEC phenotype is a key step in the process of HF (17). In the process of inflammation and tissue repair following chronic liver injury, HSCs lose their droplets (18). Various signals lead to the activation of resting HSCs and transformation into myofibroblasts (MFBs), which increases the expression of a-smooth muscle actin ( $\alpha$-SMA), thereby enhancing contractility and hardening tissue $(19,20)$. Activated HSCs are the main source of stromal MFBs; they lead to an imbalance between ECM synthesis and degradation (21). Intercellular interactions among non-parenchymal hepatocytes results in HSC activation and HF (22). Additionally, activated KCs produce various cytokines, resulting in neovascularization and an increase in intra-sinusoid pressure (23). Autocrine and paracrine signals among non-parenchymal hepatocytes regulate the progression of HF in unison, as presented in Fig. 1.

\section{LSECs regulate the hepatic microenvironment, activate HSCs and promote HF}

LSECs are the first cell type to be affected by various etiologies in the process of HF (24). They serve a key role in hepatic sinusoidal capillarization, angiogenesis and vasoconstriction, and participate in the process of HF in three key ways $(8,17,25)$.

Direct secretion of ECM by LSECs aggravates tissue hypoxia. After liver injury, LSECs acquire a fibrogenic phenotype and participate in HF through the direct secretion of ECM (24). Capillarization of the hepatic sinusoids results in a narrowing of the sinusoidal lumen and hypoxia (26). Under hypoxic conditions, LSECs are hypothesized to produce ECM (27). Furthermore, LSECs from fibrotic animals have resulted in the upregulation of type I collagen mRNA, which is 5-fold higher than that of the normal liver (28). They also secrete a large number of components to form an organized BM, including type IV collagen, laminin, nidogen, entactin and perlecan (27). Meanwhile, increasing studies have revealed the pivotal role of hypoxia in the activation of HSCs. Hypoxia induces the activation of HSCs by different mechanisms, including the transforming growth factor (TGF)- $\beta$, AMPK-mTOR, PKC $\theta$ and PVT1 oncogene-microRNA-152-autophagy-related protein 14 signaling pathways (29-31). Activated HSCs produce excessive fibrotic mediators, such as $\alpha$-SMA, type I collagen, matrix metalloproteinase (MMP)-2 and TGF receptors, which promote the abnormal accumulation of ECM (26). During the development of HF, there is an abnormal increase in the formation of collagen, as well as other ECM molecules, and the total collagen volume increases nearly 10 -fold (32). The massive accumulation of ECM restricts microvascular blood flow, accentuates the narrowing and deformation of hepatic sinusoids, and enhances hypoxia (26).

LSECs indirectly secrete cytokines to promote HSC activation. In addition to the direct secretion of ECM components, LSECs also indirectly contribute to HF by secreting proinflammatory and profibrogenic factors, including TGF- $\beta 1$ and platelet-derived growth factors (PDGFs), which activate HSCs and promote the synthesis of the ECM (11). TGF- $\beta 1$ is a polypeptide growth factor that participates in proliferation, differentiation, migration and apoptosis of HSCs as well as other cell types, via the TGF- $\beta$-receptor/Smads pathway (3). TGF- $\beta 1$ signaling also stimulates the transformation of HSCs into MFBs (33). It has been reported that TGF- $\beta 1$ gene transfection can lead to the rapid development of $\mathrm{HF}$ in vivo (3). The increased expression of TGF- $\beta 1$ not only recruits inflammatory cells and fibroblasts into the injured region causing subsequent HSC activation, but also induces the synthesis of certain BM proteins, including laminin, type IV collagen and entactin in LSECs (34). PDGFs are potent mitogens for HSCs (35). HSCs express PDGF receptor (R)- $\alpha$ and PDGFR- $\beta$. The PDGF family, including their ligands and receptors, serve pivotal roles in tissue repair and the formation of ECM (36,37). PDGF-B and PDGF-D strongly promote the migration, the conversion of HSCs to MFBs, ECM deposition and the modulation of the tissue inhibitor of metalloproteinase (TIMP)/MMP system (37,38). A previous study demonstrated that PDGFR expression was increased in chronic liver disease and overexpression of PDGF ligand induced fibrosis in mice (35). Deletion of PDGFR- $\beta$ on HSCs decreased the expression of $\alpha$-SMA and type I collagen, alleviating HF (39). Furthermore, the selective blocking of PDGFR in HF has been found to reduce the proliferation, survival and migration of activated HSCs (35). LSECs stimulate HSCs migration and recruitment to sinusoids by secreting PDGF ligands and TGF- $\beta$ (40). The enhanced motility of HSCs can facilitate their coverage around the hepatic sinusoids (41). In addition, it has been reported that PDGF promotes the angiogenesis phenotype of HSCs, regulating vascular tube formation and increasing the coverage of hepatic sinusoids, thus affecting the permeability and pressure of hepatic sinusoids (25).

In addition to TGF- $\beta$ and PDGFs, fibronectin-splice variant containing extra domain A (Fn-EDA) induce the trans-differentiation of HSCs to MFBs, which takes place in the initial stages of HF (27). In vitro studies have revealed that Fn-EDA promotes the differentiation of proto-myofibroblasts into MFBs $(27,42)$. Furthermore, the increased expression of $\alpha$-SMA, which is the cellular marker of MFBs. TGF- $\beta$ activation of $\alpha$-SMA expression results from the cooperation of different signal transduction pathways raised respectively by TGF- $\beta$ and Fn-EDA (43). It has been demonstrated that TGF- $\beta$ acts on LSECs to upregulate the rapid production of Fn-EDA (43). Although HSCs produce Fn-EDA, LSECs are the first responders and therefore may serve a key role in the early stages of HF (27).

LSECs regulate the vascular tension of hepatic sinusoids via paracrine action. LSECs regulate vascular tone by secreting certain vasodilators or vasoconstrictors, such as endothelin 1 (ET-1), thromboxane A2, cyclooxygenase 1 and nitric oxide 


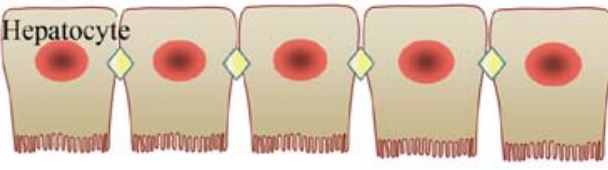

Space of disse

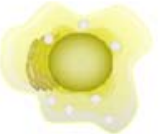

Quiescent HSC

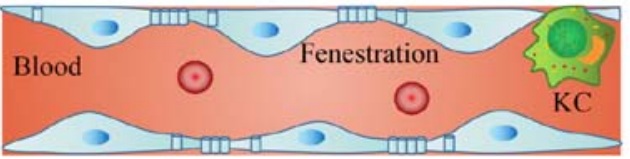

Fenestrated LSEC

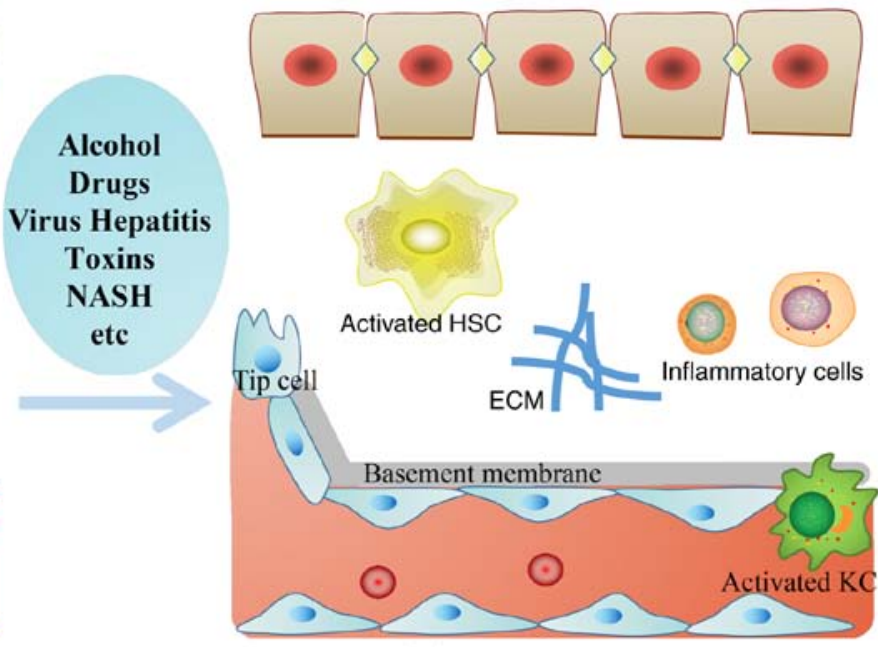

Capillarized LSEC

Figure 1. Hepatic sinusoids microenvironment alterations are induced by various chronic liver injuries. In the process of hepatic fibrosis, the morphology and function of LSECs, HSCs and KCs are abnormal, forming the subendothelial basement membrane, and decreasing endothelial cell fenestrations. HSC and KC cause a series of inflammatory responses, ECM deposition and angiogenesis. HSCs, hepatic stellate cells; KCs, Kupffer cells; ECM, extracellular matrix; LSEC, liver sinusoidal endothelial cells; NASH, non-alcoholic steatohepatitis

(NO), all of which can act on HSCs to regulate their contraction, thus regulating hepatic sinusoidal pressure (25). For example, ET-1 belongs to a family of potent vasoconstrictor peptides and is produced by LSECs or activated HSCs in a paracrine or autocrine manner (44). It was originally identified from endothelial cells (45). Elevated serum ET-1 levels have been identified in all stages of HF (45). In rat livers, the ET receptor is detected in all cell types, but most abundant in HSCs. Under normal conditions, HSCs express both endothelin A receptors (ETARs) and endothelin B receptors (ETBRs) (45). In the process of cirrhosis, ET-1 may have a stronger effect on HSCs by enhancing ETARs and ETBRs, resulting in increased microvascular tone in the hepatic sinusoids (46). ET-1 has also been revealed to upregulate the expression of procollagen I and TGF- $\beta 1$ through ETARs (47), suggesting that the biological effects of ET-1 on HSCs include not only increasing intra-sinusoidal pressure via the activation of mitogen-activated protein kinases, increased $\mathrm{Ca}^{+}$influx and reversible cell contraction, but also by increasing collagen synthesis and secretion (Fig. 2) (48).

\section{Activated HSCs interact with LSCSs to regulate the hepatic microenvironment}

The interaction between LSECs and HSCs is crucial for maintaining the structure and function of hepatic sinusoids (49). HSCs interact with LSECs in a bidirectional manner (49). As aforementioned, LSECs produce signals that activate HSCs $(17,25)$, which contributes to the capillarization of LSECs (24). HSCs promote the loss of LSEC fenestrations and the formation of continuous BM through inflammatory factors, cytokines, chemokines and vasoactive mediators (50). HSCs also induce angiogenic vascular tissue remodeling and increase vascular resistance $(51,52)$.

Capillarization of hepatic sinusoids is partially associated with the accumulation of ECM. In response to liver injury, quiescent HSCs transform into MFBs, which are the primary producers of ECM (53). MMPs serve a crucial role in ECM degradation, which is antagonized by TIMPs. Therefore, the homeostasis of ECM is coordinated by its secretion and degradation, the latter of which is partially determined by the balance between MMPs and TIMPs (54). When HSCs are activated, the expression of type I collagen, $\alpha$-SMA and TIMP-1 are upregulated, and the expression of MMP-2 is downregulated, reversing the TIMP-1/MMP-2 ratio and resulting in excessive ECM deposition and HF (55).

Under normal conditions, the hepatic sinusoids lack BM. In chronic liver disease, activated HSCs in the space of Disse produce various ECM components, including collagen IV, laminin and perlecan (27). Type IV collagen, laminin, nidogen and perlecan are the major components of BM $(56,57)$. The network of type IV collagen and laminin is linked by nidogen, which is hypothesized to be the main driving force for BM assembly (58). The co-distribution of type IV collagen and laminin is a histochemical marker of BM formation in liver disease (59). With the increase of ECM deposition in hepatic sinusoids, the number of LSEC fenestrations decreases (60). Along with BM formation, LSECs lose their fenestrae and transform into vascular type endothelium. These changes lead to hepatic sinusoidal capillarization, which seriously impedes hepatic function (59). The capillarization of LSECs is also characterized by the decrease of LSEC defensive function and the increase of platelet endothelial cell adhesion molecule-1 and Laminin 1 protein surface expression (61). The physical barrier produced by the newly formed BM impairs the intercellular exchange between parenchymal hepatocytes and the lumen (62).

In addition to ECM oversecretion, the off switch of certain signals that maintain hepatic microenvironment homeostasis in activated HSCs and LSECs contribute to the capillarization of hepatic sinusoids (63). In the healthy liver, physiological shear stress can activate the transcription factor Krüppel-like factor 2 in exposed LSECs, resulting in the increase of 


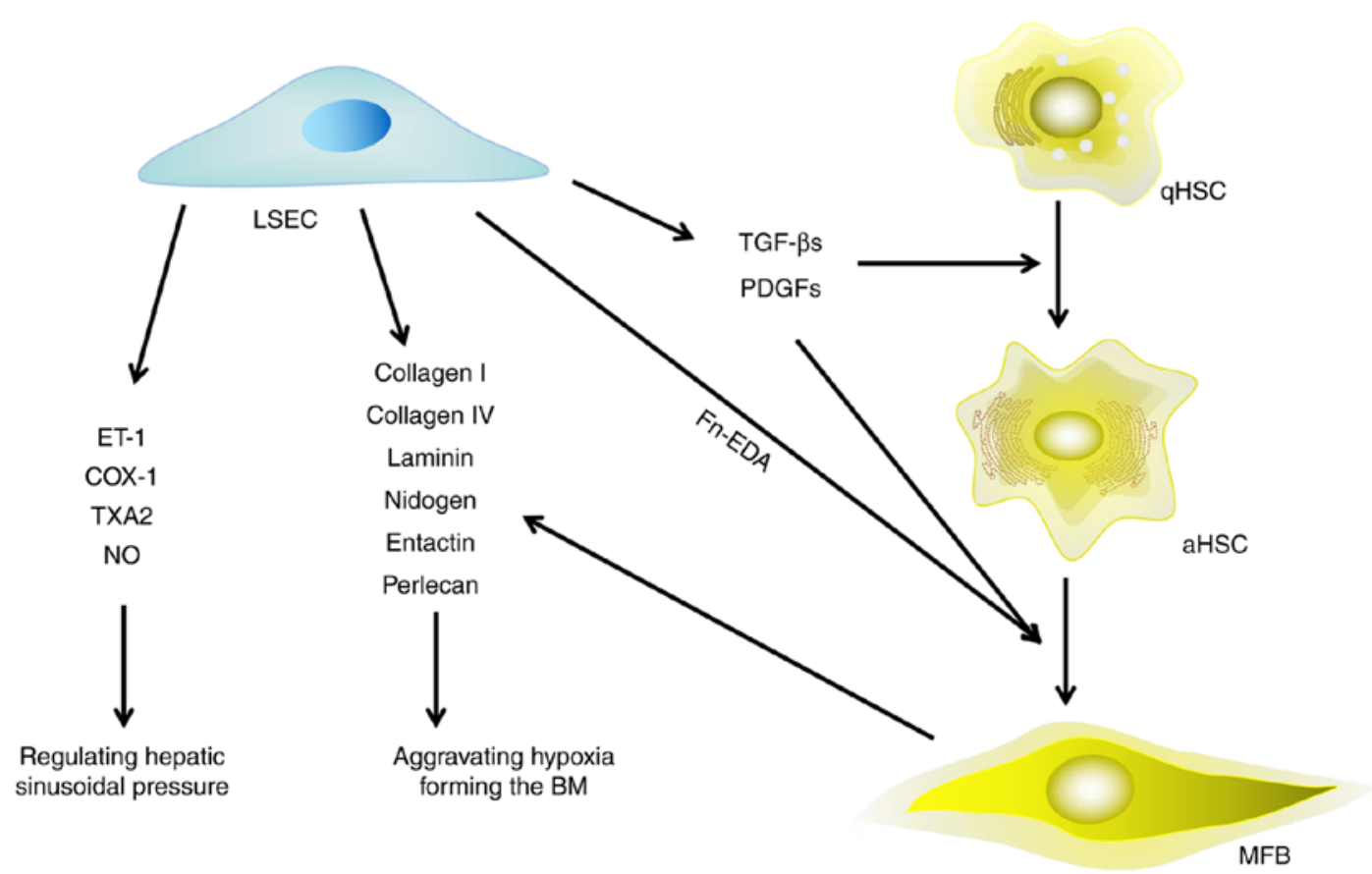

Figure 2. Effect of LSECs on HSCs, hepatic microenvironment and HF. LSECs are involved in the process of HF in three aspects: i) Secreting extracellular matrix directly; ii) secreting growth factors to promote the activation of HSCs and their trans-differentiation to myofibroblasts; and iii) releasing vasoconstrictors to regulate the pressure of hepatic sinusoids. LSECs, liver sinusoidal endothelial cells; HSCs, hepatic stellate cells; HF, hepatic fibrosis; qHSCs, quiescent hepatic stellate cells; aHSCs, activated hepatic stellate cells; MFB, myofibroblast; ET-1, endothelin 1; COX-1, cyclooxygenase 1; TXA2, thromboxane A2; NO, nitric oxide; Fn-EDA, fibronectin-splice variant containing extra domain A; BM, basement membrane; TGF- $\beta$, transforming growth factor- $\beta$; PDGF, platelet derived growth factor.

vasodilators, including $\mathrm{NO}$, and the downregulation of vasoconstrictor molecules, including ET-1, thereby alleviating the intra-sinusoidal pressure, reducing the shear stress and avoiding further injury (8). Bone morphogenetic protein 9 is a paracrine factor produced by HSCs, which maintains the quiescent state of LSECs, as well as their fenestration and terminal differentiation by binding to activin receptor like kinase 1 (64). In the process of HF, LSECs lose their aforementioned defensive signals, resulting in their impaired ability to degrade ECM, as well as the reduction of fenestrations and the formation of a continuous BM (11).

HSCs regulate the contraction of hepatic sinusoids. HSCs belong to hepatic sinusoidal pericytes and are in close contact with LSECs, meaning that their contractility regulates the contraction of hepatic sinusoids and portal vein pressure $(52,65)$. A single HSC can surround up to four individual sinusoids, and when activated, can extend itself to cover these cells (5). Previous studies have demonstrated that junctional adhesion molecule-C (JAM-C) expressed in HSCs interacts with junctional adhesion molecule-B (JAM-B) expressed in LSECs $(66,67)$. The enhanced contractibility of HSCs, which is modulated by JAM-C/JAM-B interaction, contributes to hepatic sinusoidal vasoconstriction, thereby increasing hepatic sinusoidal resistance and subsequently inducing portal hypertension (66). C-X-C chemokine receptor 4 (CXCR4) is also expressed in activated HSCs, which combines with C-X-C motif chemokine ligand 12 (CXCL12), inducing HSCs to enhance the production of type I collagen and $\alpha$-SMA (68). Moreover, CXCL12 promotes HSC migration, chemotaxis, contraction and the phosphorylation of myosin light chain in a Rho kinase-dependent manner (68). The latter is necessary for actin stress fiber assembly, as well as HSC contraction and chemotaxis (69). Vasodilators, including ET-1 and NO, are involved in the regulation of HSC contraction after activation of the Rho/rock signaling pathway (65).

HSCs participate in pathological angiogenesis. HSCs produce various biological molecules to co-regulate LSEC capillarization and angiogenesis, including vascular endothelial growth factor (VEGF), placental growth factor (PLGF) and angiopoietin (Ang) $(56,65,70)$.

VEGF is a key regulator of angiogenesis. VEGF, as well as its receptor VEGFR1, are reported to be expressed in hypoxia-stimulated activated HSCs (71). Overexpression of VEGF promotes HF by secreting ECM, whereas inhibition of either VEGFR-1 or VEGFR-2 significantly attenuates HF and angiogenesis (72). VEGF disrupts the balance of intercellular contact at the adhesion junction, enhancing permeability and leading to BM formation (50). VEGF can also stimulate LSEC proliferation and migration (73). In a normal liver microenvironment, differentiated LSECs suppress HSC activation and convert activated HSCs to the quiescence state through enhanced NO production induced by VEGF. However, when LSECs are de-differentiated or capillarized, they lose this effect $(74,75)$. Additionally, LSEC fenestration is maintained by the paracrine secretion of VEGF by HSCs and its own production of NO (63). Therefore, VEGF maintains endothelial fenestrations under physiological conditions. However, under pathological conditions, VEGF promotes the microcirculation disorder and the capillarization of hepatic sinusoids, resulting in dysfunction and structural changes in hepatic sinusoids (50). 


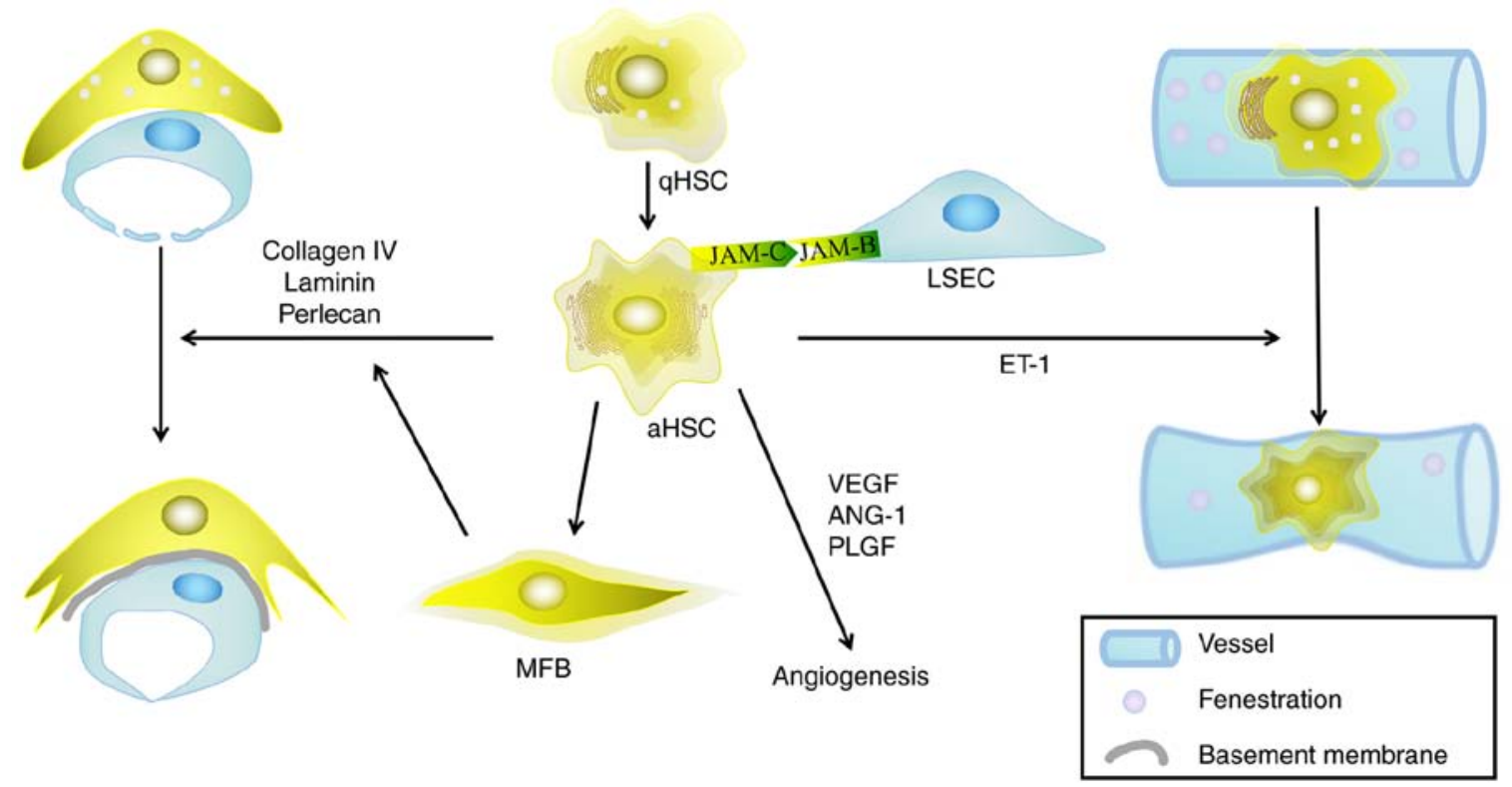

Figure 3. Activated HSCs interact with LSECs to regulate the hepatic microenvironment. HSCs are activated by various liver injuries, during which stationary HSCs are transformed into activated HSCs, leading to the remodeling of hepatic sinusoids through a series of steps. The interaction between HSCs and LSECs mainly manifest in the following ways: i) The secretion of extracellular matrix reduces the number of fenestrations in the LSECs; ii) HSCs act as pericytes to regulate the pressure of hepatic sinusoids; and iii) HSCs secrete a series of mediators that regulate angiogenesis. HSCs, hepatic stellate cells; LSECs, liver sinusoidal endothelial cells; qHSC, quiescent hepatic stellate cell; aHSC, activated hepatic stellate cell; MFB, myofibroblast; VEGF, vascular endothelial growth factor; ANG, angiopoietin; PLGF, placental growth factor; ET-1, endothelin 1; JAM-, junctional adhesion molecule.

PLGF is a member of the VEGF family and a specific ligand of VEGFR1. It was first isolated from human placenta and was demonstrated to participate in pathological angiogenesis during chronic liver disease (76). It has been demonstrated that PLGF levels are increased in patients with cirrhosis and $\mathrm{CCl}_{4}$.induced cirrhotic mice, and are mainly distributed in activated HSCs and macrophages (77). PLGF also promotes hepatic inflammation, angiogenesis and HF by mediating macrophage recruitment and activation (78). PLGF induces HSC activation and proliferation via the phosphatidylinositol 3-kinase/Akt signaling pathway (79). Furthermore, PLGF silencing can effectively alleviate inflammation and HF, inhibiting the activation of HSCs (76). Suppression of PLGF activity also alleviates the severity of HF, inflammation and portal hypertension (80). PLGF may therefore serve as a novel target for HF therapy.

The Ang/tyrosine kinase with immunoglobulin-like and EGF-like domains (TIE) signaling pathway participates in vascular homeostasis (81). Additionally, Ang family members, including Ang-1 and Ang-2, regulates angiogenesis. Their receptors, TIE- 1 and TIE-2, are tyrosine kinase that are expressed on LSECs. Ang-1 secreted by HSCs can bind to TIE-2, stabilizing newly formed vessels (26). TIE-1 maintains the structural integrity of endothelial cells, while TIE-2 is involved in angiogenesis (26). Ang-1 and Ang-2 also modulate vascular stability and vascular permeability by competitively binding to TIE-2 receptors (Fig. 3) (82).

\section{Macrophages serve a dual role in $\mathrm{HF}$ and participate in pathological angiogenesis}

Previous studies have indicated the significant role of macrophages in the regulation of HF (83-85). Macrophages are myeloid-derived immune cells that can engulf and degrade dead cells or foreign substances and coordinate the inflammatory process in response (86). Macrophages not only secrete various cytokines and growth factors, which act on HSCs and LSECs to induce the progression and regression of hepatic fibrosis (23), but they also promote the secretion of angiogenic factors, thus promoting neovascularization (87).

KCs interact with HSCs and other non-parenchymal cells to aggravate $H F$. Intrahepatic macrophages include $\mathrm{KCs}$ and infiltrating macrophages (IMs). KCs are the dominant type that aggravate liver injury and fibrosis by mediating inflammatory response (88).

KCs interact closely with other non-parenchymal cells in hepatic sinusoids and mutually interact with HSCs (89). When the liver is damaged, the number of macrophages increases dramatically, and they rapidly secrete various proinflammatory cytokines and chemokines, including interleukin (IL)-1, IL-17, tumor necrosis factor- $\alpha$ (TNF- $\alpha$ ), TGF- $\beta$, C-C chemokine ligand (CCL)-2 and CCL-5, leading to the paracrine activation of HSCs $(90,91)$. HSCs express vascular cell adhesion molecular-1, intercellular adhesion molecule (ICAM)-1, E-selection and other adhesion molecules to recruit KCs, thereby infiltrating macrophages and circulating monocytes (92).

ILs are a group of cytokines secreted by a broad spectrum of cells, including CD4-positive T lymphocytes, monocytes, macrophages and endothelial cells (91). Additionally, KCs and LSECs produce ILs rapidly after injury. Previous studies have demonstrated that mRNA levels of IL-1, IL-6 and TNF are significantly increased in isolated KCs 2-4 weeks after $\mathrm{CCl}_{4}$ injection in rats (93). ILs serve a complex role in the immune response, inflammatory reaction and HF (91). 
They directly induce the activation of HSCs and upregulate the production of TIMP-1, resulting in ECM deposition and HF (90). In addition to the direct stimulation of ECM secretion, macrophages may promote the survival of HSC via the NF- $\kappa B$ signaling pathway induced by IL-1 (94). Via NF- $\kappa B$ signaling, macrophages also increase the resistance of HSCs to cell death, thus promoting the persistence of activated HSCs and HF (90). KCs can also convert HSCs into MFBs by secreting IL-17 (95). The expression of IL-17 and its receptor IL-17RA are increased in HF mice (96). IL-17 activates NF- $\mathrm{KB}$ and signal transducer and activator of transcription (STAT) 3 signaling in KCs and HSCs (97). IL-17 also induces the transformation of HSCs to MFBs through the upregulation of TGF- $\beta 1$, TNF- $\alpha$ and type I collagen $\alpha$ subunit, which is dependent on the STAT3 signaling pathway (98).

Additionally, macrophages can release a large quantity of VEGF and induce angiogenesis during wound healing, inflammation and tumorigenesis (99). They secrete chemokine CXCL16 to recruit natural killer T (NKT) cells, thus aggravating the inflammatory response. NKT cells also promote fibrogenesis in a CXCR6-dependent manner (89).

Macrophages contribute to the termination of $\mathrm{HF}$. Macrophages serve an important role in the progression and resolution of HF (100). MFBs are generally considered to be the 'master mediators' of fibrosis, as they are the main cell types that synthesize ECM components (83). Additionally, previous studies have verified that macrophages serve an important role as the 'major regulator' of MFBs function (83). Macrophages secrete TGF- $\beta 1$, PDGF and other fibrogenic mediators to recruit and activate HSCs, as well as inflammatory cells to transform HSCs to MFBs $(83,85)$. Macrophages derived from Ly- $6 \mathrm{C}$ monocytes, which accumulate during liver injury, and mediate the transformation of HSCs into MFBs (85). Fibrogenic macrophages can transform into antifibrotic macrophages under low expression of Ly-6C and high expression of anti-inflammatory mediators (23). Antifibrotic macrophages not only remove debris directly, but also secrete factors that stimulate the production of MMPs, including gelatinases matrix metalloproteinase (MMP2 and MMP9), metalloelastase (MMP12), matrilysin (MMP7) and collagenases (MMP1 and MMP13), all of which are involved in matrix degradation, thus contributing to the resolution of fibrosis $(79,96)$. Macrophage-mediated changes in the ECM also induces apoptosis and represses the survival of MFBs, thereby reducing the ECM synthesis and contributing to the termination and even reversion of $\mathrm{HF}$ (85).

It could therefore be concluded that macrophages serve a dual role in HF and that fibrogenic macrophages contribute to the progression of fibrosis. However, anti-inflammatory macrophages are essential sources of MMPs. Therefore, macrophages function differently depending on the stage of the injury, either aggravating fibrosis or facilitating the resolution $(13,101)$.

Macrophages stimulate LSECs during angiogenesis. Fibrosis and angiogenesis may occur in parallel in multiple diseases as they share a common process with ECM accumulation and fiber formation (23). KCs are phagocytes that reside at the sinusoidal side of the endothelium. They capture signals from the blood and are involved in regulating blood flow (85). KCs and LSECs form the first barrier of the portal vein system. $\mathrm{KCs}$ are located along the neovascularization and secrete cytokines, ILs and growth factors, including VEGF, PLGF and PDGF, promoting LSEC and HSC proliferation and migration, thereby inducing pathological angiogenesis $(22,83)$.

$\mathrm{KCs}$ not only regulate vascular tension and growth through paracrine signaling during HF, but they also recruit IMs into the surrounding spouting spots by releasing monocyte chemoattractant protein-1 and TNF- $\alpha$, enhancing their pro-angiogenesis activity by multiplying their population (102). ICAM- $1 /$ macrophage-1 antigen (Mac-1)-mediated IM adhesion promotes LSEC proliferation (103). Additionally, the interaction between IMs and LSCEs though ICAM-1/Mac-1 promotes vascular endothelial (VE)-cadherin phosphorylation at the specific sites that control the passage of leukocytes, which delivers growth factors to stimulate vascular sprouting (102). IMs can also activate tyrosine-protein kinase Met or TIE-2 pathways in LSECs, which are independent of adhesion molecules, to regulate angiogenesis $(102,104)$.

Angiogenesis is initiated by a single leader endothelial cell called the 'tip cell,' which is followed by vessel elongation by 'stalk cells' (105). Endothelial tip cells are located in the front end of vascular branches, which are highly polarized and extend via a large number of pseudopodia to explore the potential environment from which they migrate, thereby stimulating angiogenesis (106). IMs can stimulate the proliferation of tip cells by releasing Wnt family member $5 \mathrm{~A}$ (Wnt5a) and stimulating the proliferation of selected stem cells by releasing Notch-1 or Ang-1 (102). IMs co-localize with Wnt5a, Ang-1 and Notch-1 at the contact point, which corresponds to the phosphorylation level of VE-cadherin and the degree of adherin-mediated intercellular junction uncoupling, driving endothelial tip cell migration and elongation (102).

To conclude, by abundant secretion of angiogenic factors, macrophages broadly participate in angiogenesis (Fig. 4).

\section{Other non-parenchymal hepatocytes also contribute to $\mathrm{HF}$}

Non-parenchymal hepatocytes include HSCs, LESCs and $\mathrm{KCs}$ as aforementioned, in addition to natural killer (NK) cells, NKT cells, dendritic cells (DCs) and biliary epithelial cells (BECs), all of which have been reported to serve diverse roles in the development of liver fibrosis (107-109).

NK cells are located in hepatic sinusoids and are in close proximity to non-parenchymal cells of the liver (110). Previous studies have demonstrated that NK cells inhibit the progression of fibrosis $(110,111)$. Under various pathological conditions, the number of NK cells increases (107). In vivo and in vitro evidence has suggested that NK cells selectively kill early activated HSCs, but not quiescent or fully activated HSCs (107). Activated NK cells produce IFN- $\gamma$, which not only directly induces HSC death, but also further enhances NK cell cytotoxicity against HSCs (112). Quiescent and fully activated HSCs do not express elevated NK cell-activating ligands and are resistant to NK cell killing (107). Other studies have suggested that NK cells can kill stressed hepatocytes through natural killer group 2 member D, natural cytotoxicity triggering receptor 3 or TNF-related 


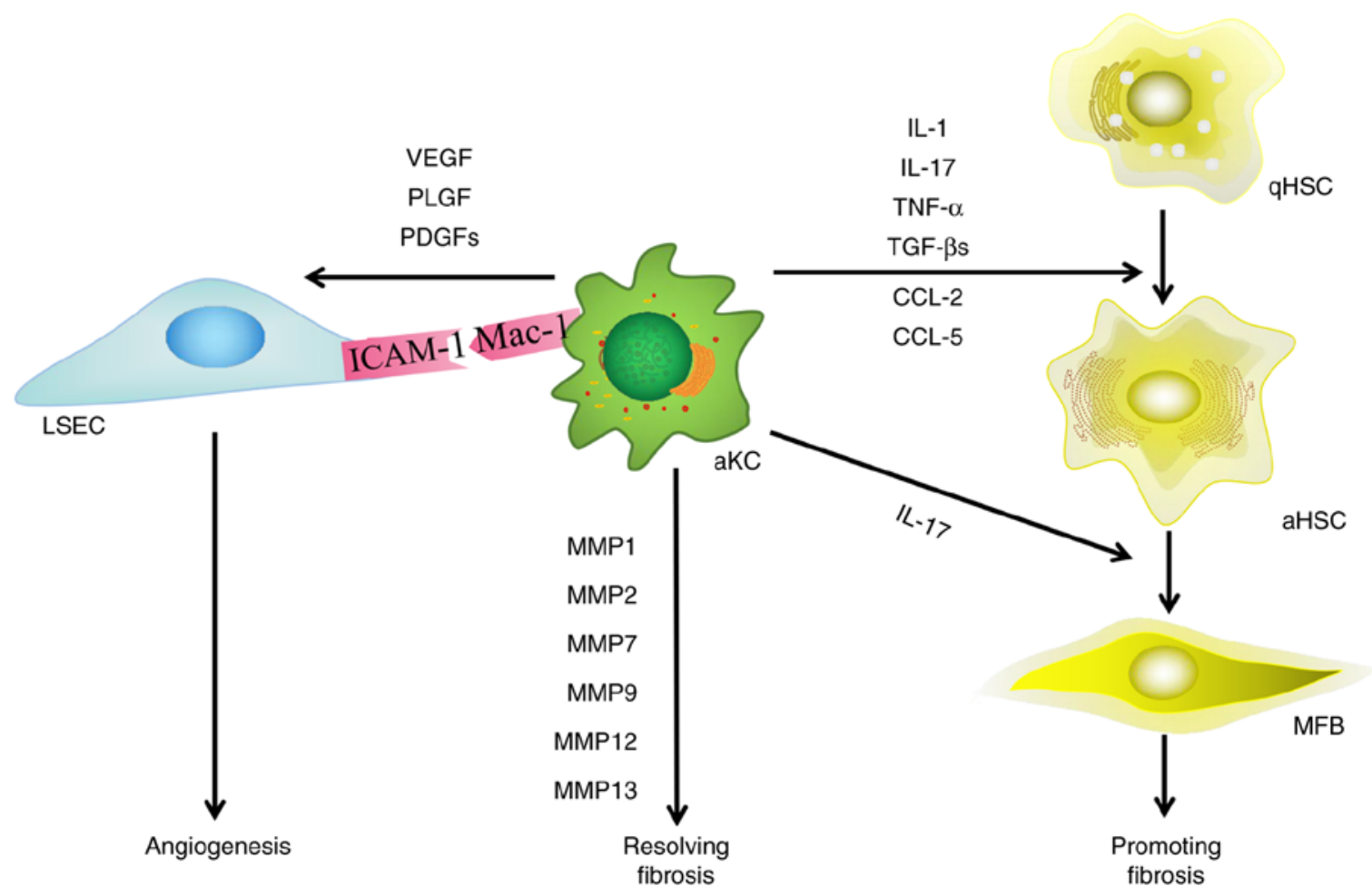

Figure 4. Effect of macrophages on HSCs and LSECs during HF. Macrophages serve a dual role in the progression and resolution of HF, and participate in angiogenesis. Macrophages activate HSCs via ILs and growth factors. When resolving HF, KCs secrete MMPs and participate in the degradation of extracellular matrix. Macrophages also induce pathological angiogenesis. HSCs, hepatic stellate cells; LSECs, liver sinusoidal endothelial cells; HF, hepatic fibrosis; VEGF, vascular endothelial growth factor; PLGF, placental growth factor; PDGF, platelet-derived growth factor; MFB, myofibroblast; aHSC, activated hepatic stellate cell; qHSC, quiescent hepatic stellate cell; ICAM-1, intercellular adhesion molecule-1; Mac-1, macrophage-1 antigen; KC, killer cells; IL, interleukin; MMP, matrix metalloproteinase; TNF- $\alpha$, tumor necrosis factor- $\alpha$; TGF- $\beta$, transforming growth factor- $\beta$; CCL-, C-C chemokine ligand.

apoptosis-inducing ligand-dependent pathways, and aggravate liver damage $(112,113)$.

In addition to NK cells, NKT cells are also enriched among liver lymphocytes (107). NK cells are important for killing virally infected or transformed hepatocytes, while NKT cells are capable of rapidly aggravating inflammatory responses under damaging conditions (94). The function of NKT cells in the pathogenesis of liver fibrosis appear more complex than NK cells. NKT cells not only produce pro-fibrotic cytokines [such as IL-4, IL-13, hedgehog (Hh) ligands and osteopontin] to promote liver fibrosis, but also produce anti-fibrotic cytokines (such as IFN- $\gamma$ ) to inhibit hepatic fibrosis (107).

DCs are professional antigen-presenting cells (92). After liver injury, DCs gain the capacity to induce inflammation via HSCs and NK cells. Previous studies have demonstrated that DCs induce HSC proliferation and produce various cytokines and chemokines, such TNF- $\alpha$ and IL-6 $(108,114)$. DCs have also been found to induce NK cell-mediated cytotoxicity in vitro by producing TNF- $\alpha$ in the livers of mice (108). Additionally, DCs are involved in the regression of fibrosis after liver injury by producing MMP9 (115).

BECs sense and respond to their surrounding microenvironment to maintain homeostasis (109). In bile duct ligation modeled mice, Hh ligand expression in HSCs and BECs increases, and cross-talk between MFBs and BECs is promoted by the Hh pathway (116). Activation of the Hh pathway stimulates BECs to specifically secrete CXCL16, which recruits NK cells into the portal vein, further promoting the inflammatory process (117). In addition, BECs secrete VEGF, which can activate the production of HSCs and collagen (109).

\section{Conclusions}

As the liver is cellularly diverse, HF is a dynamic, highly integrated process involving molecules, cells and tissues (118). Non-parenchymal hepatocytes and their interactions are broadly involved in events of the hepatic microenvironment, including hepatic sinusoid capillarization and the inflammatory reaction of the space of Disse in the development of $\operatorname{HF}(8,83)$.

Following an increased number of studies, the reversibility of HF has been demonstrated, and effective approaches for its reversal require urgent elucidation (119). It is broadly accepted that the activation of HSCs is the primary mechanism of HF (120). Other studies have concluded that the etiology of HF is the decisive factor in its development (121). Based on those considerations, the majority of the therapeutic strategies for $\mathrm{HF}$ focus on inhibiting the activation of HSCs and removing its etiology (24). However, it has been revealed that other non-parenchymal hepatocytes, including LSECs and macrophages also contribute to $\mathrm{HF}$, either dependent or independent on HSCs activation (122). Non-parenchymal hepatocytes and their interaction are broadly involved in events of the hepatic microenvironment, including hepatic sinusoids capillarization and inflammatory reaction in the space of Disse during $\mathrm{HF}$ development. Therefore, targeting one aspect of this complex and dynamic process may not be enough to achieve its reversal (1). 
In the future, the maintenance of the de-differentiation phenotype of LSECs, as well the anti-fibrotic phenotype of macrophages, is worth being considered for the treatment of HF. This necessitates comprehensive analysis of the interactions of various liver cell types and how they influence the hepatic microenvironment. Further understanding of the underlying mechanisms of these events could provide novel insight into the discovery of effective antifibrotic therapies, thus reducing the incidence of portal hypertension and hepatic encephalopathy, and blocking the formation of end stage liver disease.

\section{Acknowledgements}

Not applicable.

\section{Funding}

The present study was supported by the National Natural Science Foundation of China (grant no. 81670555).

\section{Availability of data and materials}

Not applicable.

\section{Authors' contributions}

QNC collected the data, wrote the manuscript and designed the figures. YRN designed the framework of the present review and modified the manuscript. XY and JFW assisted with manuscript preparation. YRN and WBA conceived and designed the idea for this study. All authors read and approved the final manuscript.

\section{Ethics approval and consent to participate}

Not applicable.

\section{Patient consent for publication}

Not applicable.

\section{Competing interests}

The authors declare that they have no competing interests.

\section{References}

1. Aydin MM and Akcali KC: Liver fibrosis. Turk J Gastroenterol 29: 14-21, 2018.

2. Greuter T and Shah VH: Hepatic sinusoids in liver injury, inflammation, and fibrosis: New pathophysiological insights. J Gastroenterol 51: 511-519, 2016.

3. Liu X, Hu H and Yin JQ: Therapeutic strategies against TGF-beta signaling pathway in hepatic fibrosis. Liver Int 26: 8-22, 2006.

4. Higashi T, Friedman SL and Hoshida Y: Hepatic stellate cells as key target in liver fibrosis. Adv Drug Deliv Rev 121: 27-42, 2017.

5. Marrone G, Shah VH and Gracia-Sancho J: Sinusoidal communication in liver fibrosis and regeneration. J Hepatol 65: 608-617, 2016.

6. Klenerman P and Ramamurthy N: Liver sinusoidal endothelial cells: An antiviral 'defendothelium'. Gastroenterology 148: 288-291, 2015.
7. Ray K: Liver: Hepatic stellate cells hold the key to liver fibrosis. Nat Rev Gastroenterol Hepatol 11: 74, 2014.

8. Poisson J, Lemoinne S, Boulanger C, Durand F, Moreau R, Valla D and Rautou PE: Liver sinusoidal endothelial cells: Physiology and role in liver diseases. J Hepatol 66: 212-227, 2017.

9. Shetty S, Lalor PF and Adams DH: Liver sinusoidal endothelial cells-gatekeepers of hepatic immunity. Nat Rev Gastroenterol Hepatol 15: 555-567, 2018.

10. Sorensen KK, Simon-Santamaria J, McCuskey RS and Smedsrod B: Liver Sinusoidal Endothelial Cells. Compr Physiol 5: 1751-1774, 2015.

11. Ni Y, Li JM, Liu MK, Zhang TT, Wang DP, Zhou WH, $\mathrm{Hu}$ LZ and Lv WL: Pathological process of liver sinusoidal endothelial cells in liver diseases. World J Gastroenterol 23: 7666-7677, 2017.

12. Friedman SL: Hepatic stellate cells: Protean, multifunctional, and enigmatic cells of the liver. Physiol Rev 88: 125-172, 2008.

13. Dixon LJ, Barnes M, Tang H, Pritchard MT and Nagy LE: Kupffer cells in the liver. Compr Physiol 3: 785-797, 2013.

14. Dou L, Shi X, He X and Gao Y: Macrophage phenotype and function in liver disorder. Front Immunol 10: 3112, 2019.

15. Liu HL, Lv J, Zhao ZM, Xiong AM, Tan Y, Glenn JS, Tao YY, Weng HL and Liu CH: Fuzhenghuayu decoction ameliorates hepatic fibrosis by attenuating experimental sinusoidal capillarization and liver angiogenesis. Sci Rep 9: 18719, 2019.

16. Brusilovskaya K, Konigshofer P, Schwabl P and Reiberger T: Vascular targets for the treatment of portal hypertension. Semin Liver Dis 39: 483-501, 2019.

17. DeLeve LD: Liver sinusoidal endothelial cells in hepatic fibrosis. Hepatology 61: 1740-1746, 2015.

18. Tuohetahuntila M, Molenaar MR, Spee B, Brouwers JF, Wubbolts R, Houweling M, Yan C, Du H, VanderVen BC, Vaandrager AB and Helms JB: Lysosome-mediated degradation of a distinct pool of lipid droplets during hepatic stellate cell activation. J Biol Chem 292: 12436-12448, 2017.

19. Peterova E, Podmolikova L, Rezacova M and Mrkvicova A: Fibroblast growth Factor-1 suppresses TGF- $\beta$-mediated myofibroblastic differentiation of rat hepatic stellate cells. Acta Medica (Hradec Kralove) 59: 124-132, 2016.

20. Schon HT, Bartneck M, Borkham-Kamphorst E, Nattermann J, Lammers T, Tacke F and Weiskirchen R: Pharmacological intervention in hepatic stellate cell activation and hepatic fibrosis. Front Pharmacol 7: 33, 2016.

21. Ezhilarasan D, Sokal E and Najimi M: Hepatic fibrosis: It is time to go with hepatic stellate cell-specific therapeutic targets. Hepatobiliary Pancreat Dis Int 17: 192-197, 2018.

22. Cai X, Wang J, Wang J, Zhou Q, Yang B, He Q and Weng Q: Intercellular crosstalk of hepatic stellate cells in liver fibrosis: New insights into therapy. Pharmacol Res 155: 104720, 2020.

23. Ramirez-Pedraza $M$ and Fernandez $M$ : Interplay between macrophages and angiogenesis: A double-edged sword in liver disease. Front Immunol 10: 2882, 2019.

24. Lafoz E, Ruart M, Anton A, Oncins A and Hernández-Gea V: The endothelium as a driver of liver fibrosis and regeneration. Cells 9: 929, 2020.

25. Soydemir S, Comella O, Abdelmottaleb D and Pritchett J: Does mechanocrine signaling by liver sinusoidal endothelial cells offer new opportunities for the development of anti-fibrotics? Front Med (Lausanne) 6: 312, 2019.

26. Kaur S and Anita K: Angiogenesis in liver regeneration and fibrosis: 'A double-edged sword'. Hepatol Int 7: 959-968, 2013.

27. Wells RG: Cellular sources of extracellular matrix in hepatic fibrosis. Clin Liver Dis 12: 759-768, 2008.

28. Maher JJ and McGuire RF: Extracellular matrix gene expression increases preferentially in rat lipocytes and sinusoidal endothelial cells during hepatic fibrosis in vivo. J Clin Invest 86: 1641-1648, 1990.

29. Yu F, Dong B, Dong P, He Y, Zheng J and Xu P: Hypoxia induces the activation of hepatic stellate cells through the PVT1-miR-152-ATG14 signaling pathway. Mol Cell Biochem 465: 115-123, 2020.

30. Shi YF, Fong CC, Zhang Q, Cheung PY, Tzang CH, Wu RS and Yang M: Hypoxia induces the activation of human hepatic stellate cells LX-2 through TGF-beta signaling pathway. FEBS Lett 581: 203-210, 2007.

31. Jin Y, Bai Y, Ni H, Qiang L, Ye L, Shan Y and Zhou M: Activation of autophagy through calcium-dependent AMPK/mTOR and PKC $\theta$ pathway causes activation of rat hepatic stellate cells under hypoxic stress. FEBS Lett 590: 672-682, 2016. 
32. Chen W, Rock JB, Yearsley MM, Ferrell LD and Frankel WL: Different collagen types show distinct rates of increase from early to late stages of hepatitis C-related liver fibrosis. Hum Pathol 45: 160-165, 2014.

33. Ghafoory S, Varshney R, Robison T, Kouzbari K, Woolington S, Murphy B, Xia L and Ahamed J: Platelet TGF- $\beta 1$ deficiency decreases liver fibrosis in a mouse model of liver injury. Blood Adv 2: 470-480, 2018

34. Baghy K, Iozzo RV and Kovalszky I: Decorin-TGF $\beta$ axis in hepatic fibrosis and cirrhosis. J Histochem Cytochem 60: 262-268, 2012.

35. Hayes BJ, Riehle KJ, Shimizu-Albergine M, Bauer RL, Hudkins KL, Johansson F, Yeh MM, Mahoney WJ, Yeung RS and Campbell JS: Activation of platelet-derived growth factor receptor alpha contributes to liver fibrosis. PLoS One 9: e92925, 2014.

36. Ying HZ, Chen Q, Zhang WY, Zhang HH, Ma Y, Zhang SZ, Fang $\mathrm{J}$ and $\mathrm{Yu} \mathrm{CH}$ : PDGF signaling pathway in hepatic fibrosis pathogenesis and therapeutics (Review). Mol Med Rep 16 7879-7889, 2017

37. Borkham-Kamphorst E and Weiskirchen R: The PDGF system and its antagonists in liver fibrosis. Cytokine Growth Factor Rev 28: 53-61, 2016.

38. Borkham-Kamphorst E, Meurer SK, Van de Leur E, Haas U, Tihaa L and Weiskirchen R: PDGF-D signaling in portal myofibroblasts and hepatic stellate cells proves identical to PDGF-B via both PDGF receptor type alpha and $\beta$. Cell Signal 27: 1305-1314, 2015.

39. Kocabayoglu P, Lade A, Lee YA, Dragomir A, Sun X, Fiel MI, Thung S, Aloman C, Soriano P, Hoshida Y and Friedman SL: $\beta$-PDGF receptor expressed by hepatic stellate cells regulates fibrosis in murine liver injury, but not carcinogenesis. J Hepatol 63: 141-147, 2015.

40. Thabut D and Shah V: Intrahepatic angiogenesis and sinusoidal remodeling in chronic liver disease: New targets for the treatment of portal hypertension? J Hepatol 53: 976-980, 2010.

41. Lim BJ, Lee WK, Lee HW, Lee KS, Kim JK, Chang HY and Lee JI: Selective deletion of hepatocyte platelet-derived growth factor receptor $\alpha$ and development of liver fibrosis in mice. Cell Commun Signal 16: 93, 2018.

42. Serini G, Bochaton-Piallat ML, Ropraz P, Geinoz A, Borsi L, Zardi L and Gabbiani G: The fibronectin domain ED-A is crucial for myofibroblastic phenotype induction by transforming growth factor-beta1. J Cell Biol 142: 873-881, 1998

43. Gabbiani G: The myofibroblast in wound healing and fibrocontractive diseases. J Pathol 200: 500-503, 2003.

44. Bocca C, Novo E, Miglietta A and Parola M: Angiogenesis and Fibrogenesis in chronic liver diseases. Cell Mol Gastroenterol Hepatol 1: 477-488, 2015.

45. Kardum D, Fabijanic D, Lukic A, Romic Z, Petrovecki M, Bogdanovic Z, Juric K, Urek-Crncevic $M$ and Banic $M$ Correlation of endothelin-1 concentration and angiotensin-converting enzyme activity with the staging of liver fibrosis. Coll Antropol 36: 413-418, 2012.

46. Yokomori H,Oda M,Ogi M,Kamegaya Y,Tsukada N,Nakamura M and Ishii H: Enhanced expression of endothelin receptor subtypes in cirrhotic rat liver. Liver 21: 114-122, 2001.

47. Koda M, Bauer M, Krebs A, Hahn EG, Schuppan D and Murawaki Y: Endothelin-1 enhances fibrogenic gene expression, but does not promote DNA synthesis or apoptosis in hepatic stellate cells. Comp Hepatol 5: 5, 2006.

48. Pinzani M, Milani S, De Franco R, Grappone C, Caligiuri A Gentilini A, Tosti-Guerra C, Maggi M, Failli P, Ruocco C and Gentilini P: Endothelin 1 is overexpressed in human cirrhotic liver and exerts multiple effects on activated hepatic stellate cells Gastroenterology 110: 534-548, 1996.

49. Das A, Shergill U, Thakur L, Sinha S, Urrutia R, Mukhopadhyay D and Shah VH: Ephrin B2/EphB4 pathway in hepatic stellate cells stimulates Erk-dependent VEGF production and sinusoidal endothelial cell recruitment. Am J Physiol Gastrointest Liver Physiol 298: G908-G915, 2010.

50. Li G, Peng Y, Zhao T, Lin J, Duan X, Wei Y and Ma J: Plumbagin alleviates capillarization of hepatic sinusoids in vitro by downregulating ET-1, VEGF, LN, and type IV collagen. Biomed Res Int 2017: 5603216, 2017.

51. Lee JS, Semela D, Iredale J and Shah VH: Sinusoidal remodeling and angiogenesis: A new function for the liver-specific pericyte? Hepatology 45: 817-825, 2007.
52. Rockey DC: Hepatic blood flow regulation by stellate cells in normal and injured liver. Semin Liver Dis 21: 337-349, 2001.

53. Henderson NC and Iredale JP: Liver fibrosis: Cellular mechanisms of progression and resolution. Clin Sci (Lond) 112: 265-280, 2007.

54. Knittel T, Mehde M, Kobold D, Saile B, Dinter C and Ramadori G: Expression patterns of matrix metalloproteinases and their inhibitors in parenchymal and non-parenchymal cells of rat liver: Regulation by TNF-alpha and TGF-beta1. J Hepatol 30: 48-60, 1999.

55. Liu T, Xu L, Wang C, Chen K, Xia Y, Li J, Li S, Wu L, Feng J, $\mathrm{Xu} \mathrm{S}$, et al: Alleviation of hepatic fibrosis and autophagy via inhibition of transforming growth factor- $\beta 1 /$ Smads pathway through shikonin. J Gastroenterol Hepatol 34: 263-276, 2019.

56. Gupta G, Khadem F and Uzonna JE: Role of hepatic stellate cell (HSC)-derived cytokines in hepatic inflammation and immunity. Cytokine 124: 154542, 2019.

57. Marchand M, Monnot C, Muller L and Germain S: Extracellular matrix scaffolding in angiogenesis and capillary homeostasis. Semin Cell Dev Biol 89: 147-156, 2019.

58. Mokkapati S, Fleger-Weckmann A, Bechtel M, Koch M, Breitkreutz D, Mayer U, Smyth N and Nischt R: Basement membrane deposition of nidogen 1 but not nidogen 2 requires the nidogen binding module of the laminin gammal chain. J Biol Chem 286: 1911-1918, 2011.

59. Mak KM and Mei R: Basement membrane type IV collagen and laminin: An overview of their biology and value as fibrosis biomarkers of liver disease. Anat Rec (Hoboken) 300: 1371-1390, 2017.

60. McGuire RF, Bissell DM, Boyles J and Roll FJ: Role of extracellular matrix in regulating fenestrations of sinusoidal endothelial cells isolated from normal rat liver. Hepatology 15: 989-997, 1992.

61. Braet F and Wisse E: Structural and functional aspects of liver sinusoidal endothelial cell fenestrae: A review. Comp Hepatol 1: $1,2002$.

62. Natarajan V, Harris EN and Kidambi S: SECs (Sinusoidal Endothelial Cells), liver microenvironment, and fibrosis. Biomed Res Int 2017: 4097205, 2017.

63. DeLeve LD, Wang X, Hu L, McCuskey MK and McCuskey RS: Rat liver sinusoidal endothelial cell phenotype is maintained by paracrine and autocrine regulation. Am J Physiol Gastrointest Liver Physiol 287: G757-G763, 2004.

64. Desroches-Castan A, Tillet E, Ricard N, Ouarne M, Mallet C, Belmudes L, Coute Y, Boillot O, Scoazec JY, Bailly S and Feige JJ: Bone morphogenetic protein 9 is a paracrine factor controlling liver sinusoidal endothelial cell fenestration and protecting against hepatic fibrosis. Hepatology 70: 1392-1408, 2019.

65. Soon RJ and Yee HJ: Stellate cell contraction: Role, regulation, and potential therapeutic target. Clin Liver Dis 12: 791-803, 2008.

66. Hintermann E, Bayer M, Ehser J, Aurrand-Lions M, Pfeilschifter JM, Imhof BA and Christen U: Murine junctional adhesion molecules JAM-B and JAM-C mediate endothelial and stellate cell interactions during hepatic fibrosis. Cell Adh Migr 10: 419-433, 2016.

67. Hintermann E, Bayer M, Conti CB, Fuchs S, Fausther M, Leung PS, Aurrand-Lions M, Taubert R, Pfeilschifter JM, Friedrich-Rust M, et al: Junctional adhesion molecules JAM-B and JAM-C promote autoimmune-mediated liver fibrosis in mice. J Autoimmun 91: 83-96, 2018.

68. Saiman Y, Agarwal R, Hickman DA, Fausther M, El-Shamy A, Dranoff JA, Friedman SL and Bansal MB: CXCL12 induces hepatic stellate cell contraction through a calcium-independent pathway. Am J Physiol Gastrointest Liver Physiol 305: G375-G382, 2013.

69. Sohail MA, Hashmi AZ, Hakim W, Watanabe A, Zipprich A, Groszmann RJ, Dranoff JA, Torok NJ and Mehal WZ: Adenosine induces loss of actin stress fibers and inhibits contraction in hepatic stellate cells via Rho inhibition. Hepatology 49: 185-194, 2009.

70. Zhang Z, Zhang F, Lu Y and Zheng S: Update on implications and mechanisms of angiogenesis in liver fibrosis. Hepatol Res 45: 162-178, 2015.

71. Budny T, Palmes D, Stratmann U, Minin E, Herbst H and Spiegel HU: Morphologic features in the regenerating liver-a comparative intravital, lightmicroscopical and ultrastructural analysis with focus on hepatic stellate cells. Virchows Arch 451: 781-791, 2007.

72. Coulon S, Heindryckx F, Geerts A, Van Steenkiste C, Colle I and Van Vlierberghe H: Angiogenesis in chronic liver disease and its complications. Liver Int 31: 146-162, 2011. 
73. Karkkainen MJ and Petrova TV: Vascular endothelial growth factor receptors in the regulation of angiogenesis and lymphangiogenesis. Oncogene 19: 5598-5605, 2000.

74. Xie G, Wang X, Wang L, Wang L, Atkinson RD, Kanel GC, Gaarde WA and Deleve LD: Role of differentiation of liver sinusoidal endothelial cells in progression and regression of hepatic fibrosis in rats. Gastroenterology 142: 918-927, 2012

75. Deleve LD, Wang X and Guo Y: Sinusoidal endothelial cells prevent rat stellate cell activation and promote reversion to quiescence. Hepatology 48: 920-930, 2008.

76. Li X, Yao Q, Liu H, Jin Q, Xu B, Zhang S and Tu C: Placental growth factor silencing ameliorates liver fibrosis and angiogenesis and inhibits activation of hepatic stellate cells in a murine model of chronic liver disease. J Cell Mol Med 21: 2370-2385, 2017.

77. Dewerchin M and Carmeliet P: PlGF: A multitasking cytokine with disease-restricted activity. Cold Spring Harb Perspect Med 2: a011056, 2012

78. Li X, Jin Q, Yao Q, Zhou Y, Zou Y, Li Z, Zhang S and Tu C: Placental growth factor contributes to liver inflammation, angiogenesis, Fibrosis in mice by promoting hepatic macrophage recruitment and activation. Front Immunol 8: 801, 2017.

79. Reif S, Lang A, Lindquist JN, Yata Y, Gabele E, Scanga A, Brenner DA and Rippe RA: The role of focal adhesion kinase-phosphatidylinositol 3-kinase-akt signaling in hepatic stellate cell proliferation and type I collagen expression. J Biol Chem 278: 8083-8090, 2003.

80. Van Steenkiste C, Ribera J, Geerts A, Pauta M, Tugues S, Casteleyn C, Libbrecht L, Olievier K, Schroyen B, Reynaert $\mathrm{H}$, et al: Inhibition of placental growth factor activity reduces the severity of fibrosis, inflammation, and portal hypertension in cirrhotic mice. Hepatology 53: 1629-1640, 2011.

81. Augustin HG, Koh GY, Thurston G and Alitalo K: Control of vascular morphogenesis and homeostasis through the angiopoietin-Tie system. Nat Rev Mol Cell Biol 10: 165-177, 2009.

82. Gurnik S, Devraj K, Macas J, Yamaji M, Starke J, Scholz A, Sommer K, Di Tacchio M, Vutukuri R, Beck H, et al: Angiopoietin-2-induced blood-brain barrier compromise and increased stroke size are rescued by VE-PTP-dependent restoration of Tie2 signaling. Acta Neuropathol 131: 753-773, 2016

83. Wynn TA and Barron L: Macrophages: Master regulators of inflammation and fibrosis. Semin Liver Dis 30: 245-257, 2010.

84. Ma PF, Gao CC, Yi J, Zhao JL, Liang SQ, Zhao Y, Ye YC Bai J, Zheng QJ, Dou KF, et al: Cytotherapy with M1-polarized macrophages ameliorates liver fibrosis by modulating immune microenvironment in mice. J Hepatol 67: 770-779, 2017.

85. Tacke F: Targeting hepatic macrophages to treat liver diseases. J Hepatol 66: 1300-1312, 2017.

86. Varol C, Mildner A and Jung S: Macrophages: Development and tissue specialization. Annu Rev Immunol 33: 643-675, 2015.

87. You Q, Holt M, Yin H, Li G, Hu CJ and Ju C: Role of hepatic resident and infiltrating macrophages in liver repair after acute injury. Biochem Pharmacol 86: 836-843, 2013.

88. Vollmar B, Siegmund S, Richter S and Menger MD: Microvascular consequences of Kupffer cell modulation in rat liver fibrogenesis. J Pathol 189: 85-91, 1999.

89. Wehr A, Baeck C, Heymann F, Niemietz PM, Hammerich L, Martin C, Zimmermann HW, Pack O, Gassler N, Hittatiya K, et al: Chemokine receptor CXCR6-dependent hepatic NK T cell accumulation promotes inflammation and liver fibrosis. J Immunol 190: 5226-5236, 2013.

90. Tacke F and Zimmermann HW: Macrophage heterogeneity in liver injury and fibrosis. J Hepatol 60: 1090-1096, 2014.

91. Zhou WC, Zhang QB and Qiao L: Pathogenesis of liver cirrhosis. World J Gastroenterol 20: 7312-7324, 2014.

92. Koyama Y and Brenner DA: Liver inflammation and fibrosis. J Clin Invest 127: 55-64, 2017.

93. Luckey SW and Petersen DR: Activation of Kupffer cells during the course of carbon tetrachloride-induced liver injury and fibrosis in rats. Exp Mol Pathol 71: 226-240, 2001.

94. Weiskirchen R and Tacke F: Cellular and molecular functions of hepatic stellate cells in inflammatory responses and liver immunology. Hepatobiliary Surg Nutr 3: 344-363, 2014.

95. Wang J, Leclercq I, Brymora JM, Xu N, Ramezani-Moghadam M, London RM, Brigstock D and George J: Kupffer cells mediate leptin-induced liver fibrosis. Gastroenterology 137: 713-723, 2009.

96. Meng F, Wang K, Aoyama T, Grivennikov SI, Paik Y, Scholten D, Cong M, Iwaisako K, Liu X, Zhang M, et al: Interleukin-17 signaling in inflammatory, Kupffer cells, and hepatic stellate cells exacerbates liver fibrosis in mice. Gastroenterology 143: 765-776, 2012
97. Seki E and Brenner DA: Recent advancement of molecular mechanisms of liver fibrosis. J Hepatobiliary Pancreat Sci 22: 512-518, 2015.

98. Hara M, Kono H, Furuya S, Hirayama K, Tsuchiya M and Fujii H: Interleukin-17A plays a pivotal role in cholestatic liver fibrosis in mice. J Surg Res 183: 574-582, 2013.

99. Mochida S, Ishikawa K, Toshima K, Inao M, Ikeda H, Matsui A, Shibuya M and Fujiwara K: The mechanisms of hepatic sinusoidal endothelial cell regeneration: A possible communication system associated with vascular endothelial growth factor in liver cells. J Gastroenterol Hepatol 13 (Suppl 1): S1-S5, 1998.

100. Zhang CY, Yuan WG, He P, Lei JH and Wang CX: Liver fibrosis and hepatic stellate cells: Etiology, pathological hallmarks and therapeutic targets. World J Gastroenterol 22: 10512-10522, 2016.

101. Lee UE and Friedman SL: Mechanisms of hepatic fibrogenesis Best Pract Res Clin Gastroenterol 25: 195-206, 2011.

102. Melgar-Lesmes P and Edelman ER: Monocyte-endothelial cell interactions in the regulation of vascular sprouting and liver regeneration in mouse. J Hepatol 63: 917-925, 2015.

103. Hoefer IE, van Royen N, Rectenwald JE, Deindl E, Hua J, Jost M, Grundmann S, Voskuil M, Ozaki CK, Piek JJ and Buschmann IR: Arteriogenesis proceeds via ICAM-1/Mac-1-mediated mechanisms. Circ Res 94: 1179-1185, 2004.

104. Schubert SY, Benarroch A, Monter-Solans J and Edelman ER Primary monocytes regulate endothelial cell survival through secretion of angiopoietin-1 and activation of endothelial Tie2. Arterioscler Thromb Vasc Biol 31: 870-875, 2011.

105. Priya MK, Sahu G, Soto-Pantoja DR, Goldy N, Sundaresan AM, Jadhav V, Barathkumar TR, Saran U, Jaffar AB, Roberts DD, et al: Tipping off endothelial tubes: Nitric oxide drives tip cells. Angiogenesis 18: 175-189, 2015.

106. De Smet F, Segura I, De Bock K, Hohensinner PJ and Carmeliet P: Mechanisms of vessel branching: Filopodia on endothelial tip cells lead the way. Arterioscler Thromb Vasc Biol 29: 639-649, 2009

107. Gao B and Radaeva S: Natural killer and natural killer T cells in liver fibrosis. Biochim Biophys Acta 1832: 1061-1069, 2013.

108. Connolly MK, Bedrosian AS, Mallen-St CJ, Mitchell AP, Ibrahim J, Stroud A, Pachter HL, Bar-Sagi D, Frey AB and Miller G: In liver fibrosis, dendritic cells govern hepatic inflammation in mice via TNF-alpha. J Clin Invest 119: 3213-3225, 2009.

109. Ehrlich L, Scrushy M, Meng F, Lairmore TC, Alpini G and Glaser S: Biliary epithelium: A neuroendocrine compartment in cholestatic liver disease. Clin Res Hepatol Gastroenterol 42: 296-305, 2018

110. Gao B, Radaeva S and Park O: Liver natural killer and natural killer T cells: Immunobiology and emerging roles in liver diseases. J Leukoc Biol 86: 513-528, 2009.

111. Wang $\mathrm{H}$ and Yin S: Natural killer T cells in liver injury, inflammation and cancer. Expert Rev Gastroenterol Hepatol 9: 1077-1085, 2015.

112. Radaeva S, Sun R, Jaruga B, Nguyen VT, Tian Z and Gao B: Natural killer cells ameliorate liver fibrosis by killing activated stellate cells in NKG2D-dependent and tumor necrosis factor-related apoptosis-inducing ligand-dependent manners. Gastroenterology 130: 435-452, 2006.

113. Peng Y, Yang T, Huang K, Shen L, Tao Y and Liu C: Salvia miltiorrhiza ameliorates liver fibrosis by activating hepatic natural killer cells in vivo and in vitro. Front Pharmacol 9: 762, 2018

114. Cheng JT, Deng YN, Yi HM, Wang GY, Fu BS, Chen WJ, Liu W, Tai Y, Peng YW and Zhang Q: Hepatic carcinoma-associated fibroblasts induce IDO-producing regulatory dendritic cells through IL-6-mediated STAT3 activation. Oncogenesis 5: e198, 2016.

115. Jiao J, Sastre D, Fiel MI, Lee UE, Ghiassi-Nejad Z, Ginhoux F, Vivier E, Friedman SL, Merad M and Aloman C: Dendritic cell regulation of carbon tetrachloride-induced murine liver fibrosis regression. Hepatology 55: 244-255, 2012.

116. Sato K, Meng F, Giang T, Glaser S and Alpini G: Mechanisms of cholangiocyte responses to injury. Biochim Biophys Acta Mol Basis Dis 1864: 1262-1269, 2018.

117. Omenetti A, Syn WK, Jung Y, Francis H, Porrello A, Witek RP, Choi SS, Yang L, Mayo MJ, Gershwin ME, et al: Repair-related activation of hedgehog signaling promotes cholangiocyte chemokine production. Hepatology 50: 518-527, 2009.

118. Parola M and Pinzani M: Liver fibrosis: Pathophysiology, pathogenetic targets and clinical issues. Mol Aspects Med 65: 37-55, 2019. 
119. Sohrabpour AA, Mohamadnejad M and Malekzadeh R: Review article: The reversibility of cirrhosis. Aliment Pharmacol Ther 36: 824-832, 2012.

120. Poilil SS, George TR, Moon MJ and Jeong YY: Nanoparticles for the treatment of liver fibrosis. Int J Nanomedicine 12 : 6997-7006, 2017.

121. Feng R, Yuan X, Shao C, Ding H, Liebe R and Weng HL: Are we any closer to treating liver fibrosis (and if no, why not)? J Dig Dis 19: 118-126, 2018.
122. Gracia-Sancho J, Marrone G and Fernandez-Iglesias A: Hepatic microcirculation and mechanisms of portal hypertension. Nat Rev Gastroenterol Hepatol 16: 221-234, 2019.

(i) () $९$ This work is licensed under a Creative Commons EY NO ND Attribution-NonCommercial-NoDerivatives 4.0 International (CC BY-NC-ND 4.0) License. 